\title{
Two classes of auditory processing disorder (APD) in children David Moore
}

Address: Institute of Hearing Research, University Park, Nottingham, UK

from International Society on Brain and Behaviour: 3rd International Congress on Brain and Behaviour

Thessaloniki, Greece. 28 November - 2 December 2007

Published: 17 April 2008

Annals of General Psychiatry 2008, 7(SuppI I):SI0 doi:10.1186/1744-859X-7-SI-SIO

This abstract is available from: http://www.annals-general-psychiatry.com/content/7/SI/SIO

(c) 2008 Moore; licensee BioMed Central Ltd.

Building on definitions of APD that suggest problems in the perception and awareness of basic sound comparisons (e.g. temporal and spectral resolution), we have examined the ability of large samples of 6-11 year old children to perform relatively simple audiological, auditory processing, speech-in-noise and cognitive tasks. Initial analysis shows that poorly performing children usually respond inconsistently to test items in one or more of these tasks. The proportion of these 'non-compliant' children decreases dramatically with increasing age. A second, smaller group of children are 'genuine poor performers'. They respond consistently, but at a level that is outside the range appropriate for their age. We are currently examining the performance of both these groups of children on other tasks, including various measures of spatial hearing, attention, speech intelligibility and communication skills. 